\title{
Textverstehen von Textaufgaben als sprachliche Anforderung im Mathematikunterricht
}

Gesamtüberblick: In Hinblick auf Text und sprachliche Variationen, die aufgrund von Anpassung an situative Bedingungen erfolgen, ist das Textverstehen ein zentraler Gegenstand, um sprachliche Schwierigkeiten, die aufgrund der Veränderungen von Sprache erfolgen, in der Mathematik antizipieren zu können. Dahingehend ist zur Einordnung des Textverstehens eine allgemeine Betrachtung der sprachlich kommunikativen Anforderungen im Fach Mathematik notwendig (Abschnitt 5.1). Die Beschreibung des Textverstehens ist vielfältig und kann auf verschiedenen Ebenen stattfinden (Abschnitt 5.2). Es existieren unterschiedliche begriffliche Verwendungen des Textverstehens, die unterschiedliche Aspekte des Prozesses des Verstehens betreffen (Abschnitt 5.2.1). Eine Perspektive der Betrachtung des Textverstehens sind Repräsentationen des Textes und die Betrachtung von mentalen Modellen (Abschnitt 5.2.2). Darüber hinaus können die Prozesse auf Merkmalsebene analysiert werden, die für das Textverstehen notwendig sind (Abschnitt 5.2.3). Aus den Kriterien des Textverstehens können Textmerkmale identifiziert werden, die die Textschwierigkeit erhöhen und auf den Mathematikunterricht zurückbezogenen werden (Abschnitt 5.2.4).

Zur Feststellung der Textverständlichkeit haben sich verschiedene Verfahren zur Messung und Vorhersage etabliert (Abschnitt 5.3). Ein Ansatz zur Messung stellt die Lesbarkeitsforschung dar, die durch die Häufigkeit von einzelnen Textmerkmalen die Textverständlichkeit durch einen Index ableitet (Abschnitt 5.3.1). Daneben existieren weitere Ansätze, die ebenfalls Elemente von Rezipienten einbeziehen und Aspekte definieren, die für die Entwicklung eines verständlichen Textes relevant sind (Abschnitt 5.3.2). Neben der allgemeinen Betrachtung der Textverständlichkeit ist eine Spezifikation der Untersuchung der Textverständlichkeit speziell von fachlichen Texten möglich (Abschnitt 5.3.3). Aus den Prinzipien

D. Bednorz, Sprachliche Variationen von mathematischen Textaufgaben, Bielefelder Schriften zur Didaktik der Mathematik 5, https://doi.org/10.1007/978-3-658-33003-3_5 
der Messung und Vorhersage der Textverständlichkeit lassen sich zusammenfassend allgemeine Textgestaltungs- und Optimierungsprinzipien ableiten, die dazu dienen können, Texte insoweit zu verändern, dass sie den Kriterien genügen (Abschnitt 5.3.4).

In Hinblick auf die Veränderung von Texten aufgrund der Maßgabe von Textverständlichkeitsprinzipien ergeben sich sprachliche Variationen, die den Ursprungstext in eine andere (optimierte) Version verändern (Abschnitt 5.4). Zur Veränderung von Mathematikaufgaben existieren unterschiedliche Formen von Variations- bzw. Simplifizierungsstrategien, die bei der Testung mathematischer Fähigkeiten genutzt wurden (Abschnitt 5.4.1). Speziell für den naturwissenschaftlichen und mathematikdidaktischen Forschungsbereich existiert ein sprachliches Variationsmodell zur Veränderung von Aufgaben (Abschnitt 5.4.2). Die empirischen Befunde zu den unterschiedlichen Möglichkeiten von Variationsstrategien von sprachlichen Merkmalen bei mathematischen Testaufgaben zeigen ein differenziertes Bild der Effektivität solcher Maßnahmen (Abschnitt 5.4.3).

Werden die Forschungsansätze des Textverstehens in einen Zusammenhang mit den empirischen Ergebnissen und den in Kapitel 3 und Kapitel 4 erörterten Theorien gebracht, ergeben sich offene Fragestellungen, die die Zielsetzung dieser Arbeit motivieren (Abschnitt 5.5).

\subsection{Allgemeine sprachlich-kommunikative Anforderungen im Fach}

Wie in Kapitel 2 ausgeführt, sind auch für das Fach Mathematik sprachlichkommunikative Anforderungen ein essenzieller Bestandteil des Lehrens und Lernens. Gleichzeitig nimmt das Bewusstsein von Lehrkräften in Bezug auf die sprachlich-kommunikativen Anforderungen zu, wie beispielsweise über die steigenden Anfragen zu Fortbildungen abgeleitet werden kann (Prediger, 2017).

Nach Vollmer und Thürmann (2010) werden die sprachlich-kommunikativen Anforderungen für den Mathematikunterricht insbesondere durch die Verwendung von Sprachhandlungsverben, den sogenannten Operatoren, verdeutlicht. Durch die Verwendung von Operatoren werden inhaltliche und kognitive Prozesse verbunden. So sind zentrale Sprachhandlungsverben das Erfassen, Benennen, Beschreiben, Erklären, Argumentieren, Bewerten und Aushandeln. Mittels der Handlungsverben wird eine transparente Möglichkeit geboten, die sprachlichkommunikativen Anforderungen einer Aufgabe darzustellen. Sie bieten weiterhin die Möglichkeit, in tabellarischer Form durch Alltagssprache erklärt zu werden; somit wird die Intention einer Aufgabe erleichtert und damit gleichzeitig 
eine vereinfachte Diagnose durch die Lehrkraft ermöglicht. Neben den Verben als besondere sprachliche Mittel, die institutionalisiert eingesetzt werden, werden darüber hinaus prozessbezogene Kompetenzen im Mathematikunterricht verlangt, die zum Teil sprachliche Anforderungen umfassen, z. B. das Argumentieren/Kommunizieren. Vollmer und Thürmann (2010) bezeichnen die Anbindung von sprachlichen Anforderungen an Kompetenzen als Maßnahme zur Sicherung der Überprüfbarkeit von Leistungsfähigkeit und Lernergebnissen auch für sprachliche Fähigkeiten.

Sowohl typische Sprachhandlungen als auch Kompetenzen mit sprachlichem Prozessbezug zeigen, dass es sich im Fach Mathematikunterricht nicht nur um fachliche Anforderungen des Lernens von mathematischen Gegenständen handelt, sondern auch um sprachliche Anforderungen, die eng mit dem Lernen der mathematischen Gegenstände in Zusammenhang stehen.

Vollmer und Thürmann (2010) bildeten durch die Analyse der unterschiedlichen Anforderungen der curricularen Vorgaben der Bildungsstandards und der Kehrlehrpläne der einzelnen Bundesländer eine Systematisierung der sprachlichen Anforderungen für den Fachunterricht ab. Die Systematisierung wurde unter der Prämisse der Differenzen der unterschiedlichen Fächerdomänen geleistet, die im Aspekt der kommunikativen Formen jedoch als vergleichbar bezeichnet werden können. Die Untersuchung der unterschiedlichen sprachlichen Anforderungen lassen sich auf vier Dimensionen des sprachlichen Handelns im Fach zurückführen. Vollmer und Thürmann (2010) bezeichnen die vier Dimensionen als Schulsprache, also die sprachliche Variation, die in Abschnitt 4.2.3 dargestellt wurde. Wie in Abschnitt 4.2.3 dargelegt, umfasst das Konzept der Schulsprache die sprachliche Variation durch die Nutzung von Sprache (Sprachhandlungen) im Kontext der Schule. Unabhängig von den Herausforderungen, die mit der Bezeichnung Schulsprache einhergehen (aufgrund der nicht vorhandenen Differenzierungen unterschiedlicher sprachlicher Register), bieten diese Dimensionen einen hinreichenden Überblick über die unterschiedlichen sprachlichen Anforderungen für den Mathematikunterricht.

1. Die erste Dimension umfasst das Feld des sprachlichen Handelns im Fachunterricht. Damit werden verschiedene Fähigkeiten subsummiert, die die Beteiligung an Interaktion, das Erschließen und Beschaffen von Informationen, das Strukturieren und Anpassen von Wissen, die Präsentation und Diskussion sowie das Reflektieren und Optimieren umfassen.

2. Die zweite Dimension beschreibt die kognitiv-sprachlichen Aktivitäten und die Diskursfunktion. Hiermit sind insbesondere die Sprach- und Denkhandlungen 
bezeichnet, die z. B. durch die Verwendung von Operatoren kenntlich gemacht sind.

3. Die dritte Dimension umfasst die fachunterrichtlichen Materialien, Textsorten, Genres und Zeichensysteme. Für den Mathematikunterricht ergeben sich in dieser Dimension spezifische Mittel. Auf der Ebene der Darstellungsmittel von mathematischen Gegenständen kann dies ein Geodreieck sein, das zur Messung von Winkeln eingesetzt wird. In Abschnitt 3.1 erfolgte die Erläuterung der speziellen Textsorten im Mathematikunterricht und der unterschiedlichen Aspekte der Verwendung. Außerdem ergibt sich für den Mathematikunterricht eine besondere Verwendung von Zeichensystemen. Dies umfasst numerische Zeichensysteme genauso wie Größen und weitere Symbole, die Verwendung finden; wie beispielsweise in Abschnitt 4.2.5 beschrieben, kann das numerische Zeichensystem als numerisches Register bezeichnet werden.

4. Die vierte Dimension beinhaltet die Textkompetenz- und Diskursfähigkeit. Die vierte Dimension gliedert sich in vier Hinsichten.

- Diskursstrategien: Fokussieren oder Elaborieren (primär beim Textverstehen)

- Textualitätskriterien: Register und Textstrukturen

- Sprachliche Mittel: Wortschatz und Grammatik

- Kommunikative Aktivität: Textverstehen und Schreiben

Entsprechend der vorgestellten Systematik ist das Textverstehen als sprachliche Anforderung von Bedeutung. Dies bedeutet, dass das Textverstehen von Mathematikaufgaben respektive mathematische Textaufgaben einen Einfluss auf das Lehren und Lernen von Mathematik in Leistungs-, aber auch Lernsituationen haben können. Dass das Textverstehen in Bezug auf Textaufgaben eine Anforderung für Lernende darstellt, zeigt eine nichtrepräsentative Umfrage von Prediger (2017). Die Umfrage zeigt, dass sich die meisten Lehrkräfte als bereits sensibilisiert gegenüber den Anforderungen von Textaufgaben zeigen, insbesondere, was den mangelnden Wortschatz von Lernenden betrifft.

\subsection{Perspektiven auf das Textverstehen}

Textverstehen ist auf curricularer Ebene eine klare Anforderung des Faches. Unklarer ist jedoch, was unter dem Begriff Textverstehen gemeint wird. Daneben existieren unterschiedliche Modelle, die Textverstehen theoretisch darstellen. Dahingehend ist die Klärung der unterschiedlichen Perspektive auf den Begriff des Textverstehens notwendig. 
Überblick (Abschnitt 5.2): Der Begriff des Textverstehens wird mit unterschiedlichen Begriffen in einen Bezugsrahmen gesetzt wie Textverständnis, Textverständlichkeit und Textschwierigkeit, die unterschiedlich verwendet werden (Abschnitt 5.2.1). Zur theoretischen Bestimmung des Textverstehens können zwei Ansätze unterschieden werden. Der erste Ansatz forciert die mentale Repräsentation des Textes, die der Lesende konstruiert und damit die Rolle des Rezipienten stärkt (Abschnitt 5.2.2). Der zweite Ansatz betrachtet stärker die Textmerkmale, die einen Einfluss auf das Textverstehen haben, wodurch ein Fokus auf Textfaktoren gelegt wird (Abschnitt 5.2.3).

\subsubsection{Begriffsklärung}

Die in Abschnitt 3.4.3 exemplarisch dargestellten Textarten für den Mathematikunterricht erklären intuitiv, dass Textverstehen nicht nur durch objektive Kriterien von Textmerkmalen wie die Komplexität der Sätze bestimmt ist (Christmann, 2004). Die Definition der Laplace-Wahrscheinlichkeit in der fachlichen (fachdidaktischen) Formulierung ist für mathematisch fachlich kompetente Lesende verständlich, für viele andere Lesende, die den Inhalt nicht kennen oder erst lernen, jedoch nicht. Einen Text zu lesen ist kein passiver Prozess, sondern stellt stets einen Prozess der Bedeutungskonstruktion dar. Der Rezipient eines Textes muss diesen Konstruktionsprozess leisten, wenn ein Text unter der Bedingung von Wissensvoraussetzungen des Rezipienten gelesen wird (Artelt et al., 2007; Groeben, 1982; Härtig et al., 2019). Gemäß Groeben (1982) und Christmann (2004) ist Textverstehen als sowohl kognitive als auch konstruktive Verarbeitung einer bestimmten Textsemantik zu verstehen, die als Postulat eines kognitiv konstruktiven aktiven Lesenden verstanden wird. Das bedeutet, dass neben Aspekten von Textmerkmalen immer auch die wechselseitige Beziehung zwischen Text und Lesenden und die individuellen Voraussetzungen des Lesenden mitgedacht werden müssen. Die Auffassung, sowohl Textmerkmale als auch den Lesenden und seine individuellen Voraussetzungen zu betrachten, kann als breiter Konsens in der wissenschaftlichen Diskussion zum Textverstehen betrachtet werden (Christmann, 2006; A. Frey et al., 2010; Groeben, 1982). Die Prozesse des Verstehens von Text sind damit in einem wechselseitigen Verhältnis zwischen Textmerkmalen und kognitiven und motivationalen Voraussetzungen zu betrachten. Nach Kintsch (2007) ist die Wechselwirkung als ein Prozess der sowohl textbezogenen als auch wissensbezogenen Konstruktion vereinigt, zu verstehen.

Aus der empirischen Perspektive ist der Vorgang des Textverstehen selbst nicht beobachtbar (Groeben, 1982; Pause, 1984). Der Vorgang des Verstehens 
kann nur durch das Beziehungsgefüge zwischen den Konzepten des Textverständnis und der Textverständlichkeit, die die Prozess- und Produktdimensionen widerspiegeln, rückgewonnen werden (Groeben, 1982; Pause, 1984). Aus dieser Beschreibung der notwendigen Parameter zur empirischen Beschreibung des Textverstehens leitet sich die Akzentuierung der Analyse ab. Das Textverstehen wird in Hinblick auf Analysen, die die Ebene des Rezipienten betrachten und damit auf die kognitiven Fähigkeiten und affektiven Bedingungen fokussieren, als Textverständnis bezeichnet (Christmann, 2004; Groeben, 1982; Pause, 1984). Solche Analyse forcieren den Prozess des Textverstehens. Ist die Akzentuierung der Analyse auf die Ebene der Textmerkmale gelegt, geht dies mit der Prämisse einher, dass die Textmerkmale als hauptsächlicher Mediator für das Textverstehen gelten. Eine solche Betonung betrachtet besonders den reibungslosen Ablauf der Decodierung und der Informationsverarbeitung. Diese Forschungsakzentuierung wird unter dem Begriff der Textverständlichkeit definiert (Christmann, 2004; Groeben, 1982; Pause, 1984).

Weitere Modelle verfeinern die Unterteilung in Textverständnis und Textverständlichkeit. Artelt et al. (2007, S. 12). betrachten vier unterschiedliche Determinanten. Die Determinanten Merkmale des Lesers/der Leserin und Aktivität des Lesers/der Leserin repräsentieren die Ebene des Rezipienten. Die Determinanten Leseanforderungen und Beschaffenheit des Textes beschreiben die textspezifischen Aspekte. Im Modell von Artelt et al. (2007) ist eine Wechselwirkung der Text- und Personenmerkmale vorausgesetzt, deren Gewichtung sich nach individuellen Spezifika unterscheiden kann.

Eine zentrale Ergänzung für die Facette des Textverstehens liefert Groeben (1982), indem der Text als Gegebenes betrachtet wird und eine Anpassung zwischen Lesenden und Textstruktur stattfinden muss. Gemäß Groeben (1982) wird die Ebene der Lesenden bzw. der Leserinstanz als veränderlich verstanden. Damit wird der Begriff Textverständnis aufseiten der Fragestellungen nach der Anpassung des Lesenden an den Text thematisiert, während die anders gerichtete Fragerichtung die Anpassung des Textes an den Lesenden ist und durch den Begriff der Textverständlichkeit erfolgt. Die dadurch geleistete Verknüpfung zwischen Textverstehen und Textanpassung liefert die theoretische Fundierung für sprachliche Variationen im Kontext des Textverstehens. Sprachliche Variationen und Textverstehen haben einen direkten Bezug zueinander. Sprachliche Variationen bzw. Anpassungen des Textes an den Lesenden sind unter dem Begriff der Textverständlichkeit zu betrachten und, wie in Kapitel 6 formuliert, ein bedeutendes Ziel der Entwicklung des Instruments zur sprachlichen Variation von mathematischen Textaufgaben. 
Neben dem Begriff des Textverstehens wird im Kontext des Verstehens von Texten in der mathematikdidaktischen und fachsprachlichen Forschungsliteratur häufig der Begriff der Textschwierigkeit, der sprachlichen Komplexität, der sprachlichen Schwierigkeit, aber auch der sprachlichen Hürden verwendet (Bamberger \& Vanecek, 1984; Groeben \& Christmann, 1989; Heine et al., 2018; Leiss et al., 2017; Prediger et al., 2015). Besonders die Textverständlichkeit steht in einem engen Zusammenhang mit der Textschwierigkeit. Dabei referiert Christmann (2004) von Textschwierigkeit auf Textverständlichkeit und verwendet beide Begriffe synonym. Entsprechend der Literatur werden diese Begriffe als quasi synonym verwendet; in Anlehnung zu den genannten Autoren werden für beide Begriffe unterschiedliche Richtungseffekte gedeutet. So verweist eine hohe Textschwierigkeit auf einen negativen Effekt auf die Verständlichkeit des Textes, während eine hohe Textverständlichkeit auf einen positiven Effekt auf die Verständlichkeit eines Textes referiert. Aufgrund der besseren Beschreibbarkeit je nach Gegenstand wird für die in Kapitel 8 präsentierten Ergebnisse der empirischen Studie ausschließlich der Begriff Textschwierigkeit genutzt.

In Anbetracht der allgemeinen Schwierigkeitsmodellierung für Mathematikaufgaben nach PISA und PALMA, die in Abschnitt 3.4.3 dargestellt wurde, wird zwischen technischen (Kalkül-)Aufgaben und Modellierungsaufgaben unterschieden (Neubrand et al., 2002; Pekrun et al., 2006; vom Hofe et al., 2002). Dabei ist davon auszugehen, dass die Textschwierigkeit (bzw. die sprachlogische Komplexität) einen Anteil an der Schwierigkeitsvarianz von Aufgaben besitzt (Blum et al., 2004). Es ist jedoch zu beachten, dass davon ausgegangen wird, dass die Textschwierigkeit im Schnitt einen geringeren Anteil an Schwierigkeitsvarianz erklärt als inhaltliche Kriterien wie die Grundvorstellungsintensität; diese kann jedoch aufgrund der Bedeutung der Sprache für verschiedene Mathematikaufgaben variieren (Blum et al., 2004; Kleine, 2004; Ufer et al., 2013).

Newman (1986) liefert für Mathematikaufgaben eine Klassifikation von drei Anforderungsstufen für sogenannte Leseverstehensaufgaben. Leseverstehensaufgaben werden als Mathematikaufgaben bezeichnet, für die das Textverstehen relevant ist. Die drei Stufen sind die folgenden:

1. Mechanische Lesefähigkeit, die das Erkennen von Wörtern und Symbolen betrifft. Daneben sind das Tempo und die Art des Lesens und das Verstehen des Kontextes Elemente.

2. Begriffsverständnis.

a. Niveau 1: Lesen, um Anweisungen zu befolgen. Dafür notwendig sind bekannte Wörter und Zahlen, um Informationen zusammenzutragen und daraus die Lösung nach einer festgegebenen Reihenfolge zu bewältigen. 
b. Niveau 2: Interpretieren. Hierfür notwendig sind Fähigkeiten zur Vorhersage von Ereignissen und die Interpretationsfähigkeit von generellen Bedeutungszuschreibungen bei gleichzeitigem Ignorieren von irrelevanten Informationen. Daneben ist das Interpretieren von Grafiken dem zweiten Niveau zugeordnet.

c. Niveau 3: Abschließend bewertende Fähigkeiten.

3. Anwendungsfähigkeit. In dieser Stufe werden das selbständige Nutzen eines Lehrbuchs und die eigenständige Formulierung von Sachverhalten subsummiert.

Nach Klärung und Beschreibung der verschiedenen terminologischen Verwendungen, die in Beziehungen zum Begriff Textverstehen stehen, werden anschließend unterschiedliche Analyseebenen des Begriffs betrachtet.

\subsubsection{Ebene der Textrepräsentation}

Besonders psychologische Ansätze fokussieren bei ihrer Betrachtung von Textverstehen Aspekte der Repräsentation des Textes. Repräsentation bedeutet, dass der Rezipient den Text bei seiner Bedeutungskonstruktion durch ein inneres mentales Bild und Beziehungsstrukturen aufbaut (Schiefele, 1996; Schnotz, 2005). Gemäß Schiefele (1996) ist von einer Distinktion zwischen Wahrnehmung und Repräsentation von Text auszugehen, wodurch die nichttextadäquate Interpretation der Intention des Autors mitinbegriffen wird. Diese Perspektive konzentriert sich bei der Ermittlung des Textverstehens darauf, die externe sprachliche Botschaft in Bezug auf die intendierte interne Textverarbeitung zu evaluieren. Textverstehen unter einer repräsentationalen Perspektive bedeutet daher die Konstruktion vielfältiger mentaler Repräsentationen, in denen der Text als vorwissensgeleitet erachtet wird und zur mentalen Modellkonstruktion, -evaluation und -revision beiträgt (Schnotz, 2005).

Nach Schnotz (2005) können unterschiedliche mentale Repräsentationsebenen unterschieden werden. Es wird unterschieden zwischen der Oberflächenebene, die die Repräsentation der Textoberfläche umfasst, der propositionalen Ebene als Repräsentation des semantischen Gehalts und der Modellebene als das mentale Modell des beschriebenen Gegenstands. Unterscheidungen lassen sich zwischen den Repräsentationsebenen sowohl in den Eigenschafen als auch in Funktionen der Repräsentationen beschreiben.

Die Oberflächenebene ist die basale mentale Repräsentationsebene und bildet damit den Aufbau für die höhere Repräsentationsebene. Die Oberflächenebene 
ermöglicht eine wortwörtliche Wiederholung von Textabschnitten, ohne dass diese zwangsläufig verstanden werden müssen (Schnotz, 2005).

Die propositionale Ebene repräsentiert den semantischen Gehalt, der aus Propositionen besteht. Die definitorische Bestimmung des Begriffs Propositionen erfordert eine differenzierte Betrachtung der unterschiedlichen Facetten des Begriffs. Dänzer und Hoeltje (2017, S. 367) fassen für Propositionen sieben Konzepte zusammen, die als grundlegend erachtet werden können:

1. Inhalte von mentalen Zuständen, z. B. Überzeugungen und Wünsche

2. Inhalte von Sprechakten, z. B. Behauptungen und Fragen

3. Träger von Wahrheitswerten

4. Bedeutungen von Deklarativsätzen

5. Bezugsobjekte von dass-Sätzen

6. Abstrakte Entitäten, z. B. in ähnlicher Weise wie Mengen und Zahlen

7. Existenziell unabhängig von geist- und sprachbegabten Wesen

Ballstaedt et al. (1981) betrachten unter der Perspektive des Textverstehens Propositionen als kleinste partikuläre Einheit, die als Satz verstanden werden kann, in einem Text vorkommt und sowohl intraindividuelle als auch strukturellsemantische und logische Zustände abbildet. Die propositionale Theorie betrachtet dabei Propositionen als Repräsentanten für Oberflächen- und Tiefenstrukturen (Kintsch \& van Dijk, 1978)

Nach Schnotz (2005) ermöglicht die propositionale Repräsentation, im Gegensatz zur Oberflächenebene, das Verstehen (Nachvollziehen) dessen, was gesagt wird, unabhängig davon, ob der Sachverhalt selbst verstanden wurde. Situationen, die diesen Bedingungen genügen, kommen in der alltäglichen Kommunikation kaum vor, sind jedoch bei hochkomplexen und schwierigen Texten nicht unüblich. Schnotz (2005) verweist auf Sätze, die zwar durch propositionale Repräsentationen abgebildet werden können, für die sich jedoch kein konkreter Sachverhalt vorgestellt werden kann. Das können Sachverhalte sein, die sich durch eine hohe Abstraktionsstufe auszeichnen und beispielsweise in der Mathematik häufig vorkommen.

Textverstehen als reine Verknüpfung von Propositionen ist jedoch nicht ausreichend oder erschöpfend, da, wie bereits oben erwähnt, das Verstehen von Texten immer auch die Bedeutungskonstruktion beinhaltet. Die Perspektive der Bedeutungskonstruktion wird besonders durch die Ebene der Repräsentation eines mentalen Modells erfüllt (Schnotz, 2005). Die Verwendung des Begriffs Mentales Modell ist jedoch umstritten. Nach Perrig und Kintsch (1985) handelt es sich bei allen erläuterten Ebenen der Textrepräsentation um mentale Modelle. 
Dahingehend wird vorgeschlagen, statt des Begriffs Mentales Modell den Begriff Situationsmodell zu verwenden, da es sich bei der letzten Ebene um die Gegenstandsebene bzw. Situationsebene handelt, in der der Text dargestellt wird (Artelt et al., 2007; van Dijk \& Kintsch, 1983).

Zur Beschreibung des Textverstehens, in Hinblick auf mentale Modelle, ist der Begriff der Fokusaktivierung bedeutsam. Laut Schnotz (2005) hat die Fokusaktivierung eine limitierende Eigenschaft für das Textverstehen, da das Arbeitsgedächtnis nur begrenzte Kapazitäten zur Verfügung hat und sich entsprechend nur begrenzt fokussieren kann. Beispielhaft für solche Aktivierungsund Fokussierungsprozesse sind mentale Modelle, die durch sogenannte TopicMarkierungen gegeben sind. Schnotz (2005) beschreibt, dass Topic-Markierungen durch pronominale und nominale Referenzen realisiert werden. Die pronominale Referenz bezieht sich durch ein Pronomen auf einen Referenzgegenstand. Damit soll dem Rezipienten deutlich gemacht werden, dass das, was zuvor explizit beschrieben wurde, noch aktuell ist und keine Fokusverschiebung stattfinden muss. Eine nominale Referenz forciert auf etwas noch nicht zwangsläufig Genanntes, es kann also eine neue Aktivierung des Fokus stattfinden. Je nachdem, welches Referenzmodell gewählt wird, können sich unterschiedliche mentale Repräsentationen ergeben. Für einzelne Fälle bietet sich eine nominale Referenz an, wenn bei weiterer Bezugnahme wieder auf das Ursprungswort zugegriffen werden kann; so können dauerhaft Oberflächenrepräsentationen, propositionale Repräsentation und mentale Modelle vorkommen. Bei der Nutzung von pronominalen Referenzen fällt die Oberflächenrepräsentation weg und dies kann zu einer schwierigeren Fokussierung führen. Zur Verdeutlichung der pronominalen und nominalen Referenz dienen die drei selbstformulierten Beispiele; die unterstrichenen Begriffe markieren die jeweiligen Referenzen:

- Beispiel eines Aufgabenteils einer Mathematikaufgabe mit pronominaler Referenz: Auf ihrer Reise nach Zürich stoppt Kerstin die Zeit und notiert, wie lange ihr Zug im Bahnhof steht [...]

- Beispiel eines Aufgabenteils einer Mathematikaufgabe mit nominaler Referenz (ohne Fokusverschiebung): Sabine und Michael machen ein Wettrennen über $100 \mathrm{~m}$. Michael lässt Sabine einen Vorsprung von 2 Sekunden [...]

- Beispiel eines Aufgabenteils einer Mathematikaufgabe mit nominaler Referenz (mit Fokusverschiebung): Frau Müller kauft Kinokarten für einen Klassenausflug für $9 €$ pro Karte. Herr Schmidt hat einen Mengenrabatt bekommen und musste nur $6 €$ pro Karte bezahlen [...] 
Für die Mathematikdidaktik ist die Betrachtung der Textrepräsentation zur Beschreibung des Textverstehens auch unter theoretischer Betrachtung interessant. So können mentale Modelle zur Beschreibung des Textverstehens genutzt werden und dienen auch in der Mathematikdidaktik dazu, innere Konstruktionsprozesse für mathematische Inhalte zu beschreiben. Damit existieren zumindest auf konzeptioneller Basis Parallelen zwischen Textrepräsentation und mathematischen Grundvorstellungen als mentale Modelle zu mathematischen abstrakten Gegenständen (Blum et al., 2004; vom Hofe, 1992; vom Hofe et al., 2005). Dass die Verknüpfung naheliegt, zeigt die in Abschnitt 3.2.5 dargestellte Einführung des Begriffs des Situationsmodells als mentale Repräsentation der Textbasis von Modellierungsaufgaben in der Mathematik (Blum \& Leiß, 2005; Leiss et al., 2010, 2019). Situationsmodelle dienen als Vermittler zwischen Realsituation und Realmodell, während Grundvorstellungen notwendig sind, um Übersetzungsprozesse zwischen realer Welt und Mathematik zu leisten.

\subsubsection{Ebene der Textprozesse}

Unter der Perspektive von prozessbezogenen Bedingungen des Textverstehens wird vom prozeduralen Aspekt gesprochen (Schnotz, 1987). Hierbei stehen Textmerkmale im Vordergrund, die zur Verarbeitung des Textes eine notwendige Voraussetzung sind. Für die Betrachtung des Textverstehens auf Textprozessebene werden Textmerkmale selektiert und es wird untersucht, in welcher Weise die sprachlichen Merkmale einen Einfluss auf den Prozess der Kommunikation und die Textverständlichkeit für den Rezipienten haben können. Dadurch ergibt sich ein Modell, das nicht nur einzelne Variablen der Textverständlichkeit betrachtet, sondern die wechselseitige Wirkung der Variablen in die Analyse integriert (Heringer, 1984).

Nach Bayer und Seidel (1979) besteht die Möglichkeit, den Textverständlichkeitsbegriff in Hinblick auf eine Konzentration auf spezifische sprachliche Merkmale eingeschränkt zu betrachten. Eine solche Vorgehensweise bedeutet, dass die Analyse von Textmerkmalen erfolgt, während andere Aspekte aus dem Kommunikationsprozess nicht betrachtet werden. Aus diesem Grund wird nachfolgend statt des Begriffs Textverstehen, der sowohl Rezipienten- als auch Textmerkmale betrachtet, der limitierende Begriff der Textverständlichkeit bzw. Verständlichkeit verwendet.

Für die Klassifikation unterschiedlicher Teilprozesse der Textverständlichkeit wird in der Forschungsliteratur der Linguistik, der Mathematikdidaktik und der Bildungsforschung meist nach Verständlichkeit auf Wort-, Satz- und 
Textebene unterschieden (Artelt et al., 2007; Czicza \& Hennig, 2011; Gürsoy et al., 2013; Heppt et al., 2014; Leisen, 2013; Prediger, 2013b; Schmitz, 2015). Diesbezüglich sollen die unterschiedlichen Ebenen inhaltlich beschrieben werden; ergänzend dazu wird in Abschnitt 5.3.2 auf die Schwierigkeiten von Textmerkmalen eingegangen, die für die unterschiedlichen Ebenen resultieren.

Verständlichkeit auf Wortebene: Der Ausgangspunkt der Verständlichkeit auf Wortebene ist die sensorische Verarbeitung von optischen Reizen (Ballod, 2001; Halliday, 2014a). Nach Artelt et al. (2007) kann die Entwicklung der Bedeutung durch die durch sensorische Wahrnehmung erfassten Buchstabenfolgen (Wörtern) als lexikalischer Zugriff bezeichnet werden. Zentraler Aspekt für die Verständlichkeit auf Wortebene ist die Verarbeitung und Erkennung von Wörtern. Dabei wird durch die Verarbeitungsprozesse das semantische Gedächtnis bzw. das mentale Lexikon für die Erkennung aktiviert (Just \& Carpenter, 1980).

Verständlichkeit auf Satzebene: Gemäß Just und Carpenter (1980) müssen die Wörter neben einer separierten Erkennung und Verarbeitung durch eine Bedeutungseinheit in einen Gesamtzusammenhang gebracht werden. Rickheit und Strohner (1983) unterscheiden die Verständlichkeit auf der Ebene des Satzes in fünf Phasen. Die Phasen umfassen:

1. Informationsaufnahme

2. lexikalische Bedeutungszuweisung

3. Bestimmung der Kasusrollen

4. Integration von Teilsätzen

5. Satzabschluss

Nach Ballstaedt et al. (1981) kann angenommen werden, dass die einzelnen Einheiten der Bedeutung durch Propositionen repräsentiert sind. Mit der Einteilung wird die syntaktische Ebene betrachtet, also der Bedeutung, die in einem Text hinterlegt ist, durch die im Text kodierten Wörter.

Verständlichkeit auf Textebene: In Bezug auf die Verständlichkeit auf der Ebene des Textes werden zum einen die bereits in Abschnitt 5.2.2 geschilderten repräsentationalen Aspekte bedeutend. Damit werden bei der Analyse auf Textebene die sprachlichen Merkmale eines Textes betrachtet, die sich als relevant für den aktiven Konstruktionsprozess auszeichnen. Im Beispiel der Modellierungsaufgabe Feuerwehr kann verdeutlicht werden, welchen Einfluss repräsentationale Aspekte auf die Verständlichkeit auf Textebene haben können. Um ein adäquates mathematisches Modell zu bilden, ist für die Modellierungsaufgabe notwendig, 
eine mentale Vorstellung davon zu bilden, was unter einem Drehleiter-Fahrzeug verstanden wird. In der Modellierungsaufgabe wird die Bildung eines Situationsmodells dadurch erleichtert, dass eine Illustration (nicht mit abgebildet) eines Drehleiter-Fahrzeugs dargestellt wird. Das bedeutet, dass einzelne Textmerkmale einen Einfluss auf die gesamte Repräsentation des Textes haben können und damit die Verständlichkeit auf Textebene beeinflussen.

Feuerwehr:

Die Münchner Feuerwehr hat sich im Jahr 2004 ein neues Drehleiter-Fahrzeug angeschafft. Mit diesem kann man über einem am Ende der Leiter angebrachten Korb Personen aus großen Höhen retten. Dabei muss das Feuerwehrauto laut einer Vorschrift $12 \mathrm{~m}$ Mindestabstand vom brennenden Haus einhalten.

Die technischen Daten des Fahrzeugs sind:

$\begin{array}{ll}\text { Fahrzeugtyp: } & \text { Daimler Chrysler AG Econic 18/28 LL - Diesel } \\ \text { Baujahr: } & 2004 \\ \text { Leistung: } & 205 \mathrm{kw}(279 \mathrm{PS}) \\ \text { Hubraum: } & 6374 \mathrm{~cm}^{3} \\ \text { Maße des Fahrzeugs: } & \text { Länge } 10 \mathrm{~m} \text { Breite } 2,5 \mathrm{~m} \text { Höhe } 3,19 \mathrm{~m} \\ \text { Maße der Leiter: } & 30 \mathrm{~m} \text { Länge } \\ \text { Leergewicht: } & 15540 \mathrm{~kg} \\ \text { Gesamtgewicht: } & 18000 \mathrm{~kg}\end{array}$

Aus welcher maximalen Höhe kann die Münchner Feuerwehr mit diesem Fahrzeug Personen retten? Schreibe deinen Lösungsweg auf (Blum und Leiss, 2007).

Weitere Ansätze zur Determination der Textverständlichkeit, neben der Unterscheidung zwischen Wort-, Satz- und Textebene, insbesondere in Hinblick auf den Begriff der Textschwierigkeit, bieten Forschungen zur Konstruktion von Sprachtests. Grotjahn (2000) bietet eine Möglichkeit, unterschiedliche Schwierigkeitsindikatoren der Textschwierigkeit zu unterscheiden, die in drei Typen von Schwierigkeitsprädikatoren untergliedert werden (vollständige Auflistung siehe Anhang).

1. Itemvariablen, bei denen beispielsweise Ambiguität der Item-Formulierung und die Anzahl der schwierigen Wörter betrachtet werden und mit der vorher diskutierten Wortebene verglichen werden können.

2. Textvariablen, hierunter fallen die Komplexität der Satzstruktur und die Zahl der referenziellen Einheiten. Diese Ebene kann mit der betrachteten Satzebene verglichen werden. 
3. Text-Item-Variablen integrieren sowohl Item- als auch Textvariablen, bei denen betrachtet wird, ob nur Hauptinformationen erfragt oder Hintergrundinformationen benötigt werden.

Die Entwicklung des Instruments zur sprachlichen Variation von Textaufgaben hat den Fokus, ausschließlich Textmerkmale in die sprachlichen Veränderungen von Textaufgaben einzubeziehen. Das Instrument soll zur Anpassung von Texten dienen, um die Textverständlichkeit unter gewissen Prämissen zu verändern. Dahingehend werden nachfolgend die für das Forschungsinteresse relevanten Einflüsse von Textmerkmalen auf die Textschwierigkeit betrachtet.

\subsubsection{Erklärungsansätze des Einflusses von sprachlichen Merkmalen und Konkretisierung auf die Mathematikdidaktik}

Wie bereits in Abschnitt 5.2.3 erörtert, beginnt der Prozess des Textverstehens mit der Erkennung und Verarbeitung von Wörtern. Gemäß Rayner und Pollatsek (1989) können Identifikationsmodelle von Wörtern interpretiert werden, als differenziellen lexikalischen Zugriff, der aufgrund von unterschiedlichen kognitiven Verarbeitungsebenen in Merkmals-, Buchstaben- und Wortebene unterschieden wird. Das Erkennen und Verarbeiten von Wörtern wird unter Betrachtung dieser kognitiven Verarbeitungsebenen betrachtet. Insbesondere Modelle für die Wortebene betrachten Worthäufigkeitseffekte. Postuliert wird, dass häufige Wörter schneller erkannt werden als Wörter, die eher selten in der jeweiligen Sprache vorkommen. Just und Carpenter (1987) zeigten in ihrer Studie zu Verarbeitungseffekten, dass Inhaltswörter (Nomen, Verben, Adjektive, Adverben) länger fixiert werden als z. B. Funktionswörter (Artikel, Präpositionen, Konjunktionen), die bei der Untersuchung teilweise übersprungen wurden. Nach Grimm und Engelkamp (1981) ist der höhere Verarbeitungsaufwand durch Textelemente wie Inhaltswörter damit zu erklären, dass Inhaltswörter im Vergleich zu Funktionswörtern weniger häufig auftreten. Die kürzere Dauer der Betrachtung von Funktionswörtern ist damit zu erklären, dass diese einen niedrigeren Gehalt an Informationen und im mentalen Lexikon zur Aktivierung ein höheres Niveau aufweisen (Forster, 1994; Underwood \& Batt, 1996). Für die Wortebene stellt die Worthäufigkeit ein potenzielles Merkmal für die Textschwierigkeit dar (Taft, 1979). Je häufiger insbesondere inhaltsgeprägte Wörter verwendet werden, die gegebenenfalls unvertraut sind, desto mehr führt dies zu Schwierigkeiten im Textverstehen. 
Für den Mathematikunterricht sind mathematische Fachbegriffe mögliche Wörter, die die Textschwierigkeit beeinflussen und entsprechend für die Wortebene als häufiges schwierigkeitsgenerierendes Kriterium in der Literatur genannt werden (Abshagen, 2015; Härtig et al., 2019; Leisen, 2013; Leisen \& Seyfarth, 2006; Schmiemann, 2011). Arya et al. (2011) konnten für den naturwissenschaftlichen Forschungsbereich zeigen, dass alltägliche Formulierungen im Vergleich zur Nutzung von Fremdwörtern einen Effekt auf die Textverständlichkeit haben. In Anbetracht von Funktionswörtern im Mathematikunterricht bzw. in mathematischen Texten sind Funktionswörter für die Konzeptualisierung mathematischer Beziehungen essenziell (Prediger, 2013b). In Hinblick auf die geringe Fixationsdauer und eine potenziell hohe Bedeutung der Funktionswörter für das Verstehen des mathematischen Inhalts in einem Text kann die Textverständlichkeit beeinflusst werden.

Neben Worthäufigkeitseffekten hat die Wortlänge einen Einfluss auf die Textverständlichkeit. Die Wortlänge bezieht sich auf die morphologische Komplexität eines Textes und ist ebenfalls als Zugriff auf das mentale Lexikon zu deuten (Feldman, 1991; Schriefers, 1999). Nach Just und Carpenter (1980) kann der Effekt der Wortlänge auf den Verarbeitungsaufwand verdeutlicht werden. So zeigt sich, dass die Fixierung von langen Wörtern deutlich höher ausfällt als die von kurzen Wörter, was durch die gestiegene Silbenanzahl erklärt wird. In dieser Hinsicht ist in der deutschen Sprache die Kompositaverwendung ein Merkmal, das Effekte auf die Textverständlichkeit zeigen kann und häufig in fachlichen und mathematischen Texten vorkommt (Abshagen, 2015; Leisen, 2013; Leisen \& Seyfarth, 2006). Feldman (1991) betrachtet Verarbeitungseffekte auf Basis von Morphemen, das bedeutet, die Flexionen und Derivationen von Wortstämmen. Durch die Betrachtung von Morphemen kann die zusätzliche kognitive Beanspruchung des lexikalischen Zugriffs modelliert werden. Die kognitive Beanspruchung ist besonders für längere und zusammengesetzte Wörter bedeutsam, weshalb die Silbenanzahl einen guten Indikator für die Beanspruchung darstellt.

Neben den Effekten, die sich auf die Worthäufig und -komplexität beziehen, werden insbesondere in der naturwissenschaftlichen Didaktik Worteffekte auf Ebene der Textkohäsion betrachtet (Starauschek, 2006). Nach Pause (1984) ist die Anaphorik ein bedeutendes Mittel zur Bildung von Textkohäsion. Mit Anaphorik sind die Verweisstrukturen, die in einem Text gebraucht werden, gemeint, die zum einen die referentielle und zum anderen die thematische Kontinuität eines Textes bilden. Dabei lassen sich unterschiedliche anaphorische Bezüge unterscheiden, die je nach Bedingungen Schwierigkeiten für die Textverständlichkeit ergeben können. Hierbei spielen u. a. die Nutzung von nominaler Wiederaufnahme (explizite Verweise), Pronomen und die synonyme Verwendung von Wörtern eine Rolle, 
deren Relevanz durch empirische Ergebnisse bestätigt und durch einen höheren kognitiven Aufwand erklärt wird (Arya et al., 2011; Ozuru et al., 2009; Starauschek, 2006). Weitere bedeutende Textmerkmale auf Wortebene zur Bildung von Verweisstrukturen, um logische und inhaltliche Zusammenhänge im Text herzustellen, sind Wiederaufnahmestrukturen, Konjunktionen und Präpositionen (Groeben \& Christmann, 1989).

Neben kognitiven Aspekten und dem Zugriff auf das mentale Lexikon können relationale Diskrepanzen zwischen Wörtern einen Effekt auf die Textverständlichkeit zeigen. Dies ist für mathematische Begriffe interessant, die im mathematischen und im alltäglichen Kontext eine gleiche oder andere Bedeutungszuschreibung besitzen (Groeben \& Christmann, 1989). In der Begriffsfindung der Domäne Mathematik bedienen sich Mathematikerinnen und Mathematiker zum Teil an bestehenden Begriffen aus der Alltagssprache, durch die eine Bedeutungsübertragung stattfindet. Gemäß Wessel (2015) dienen Fachbegriffe zur Vorstellungsentwicklung, da formalbezogene Fachsprache nicht ausreichend ist, um ein inhaltliches Verständnis zu festigen. Nach Malle (2009) kann die Verwendung von Metaphern (z. B.: Wurzel, Wurzel ziehen) als Brückenglied zur formalbezogenen Sprache dienen. Fachwörter bieten die Möglichkeit zur Differenzierung und Systematisierung inhaltlicher Konzepte. Die Vernetzung von bedeutungs- und formalbezogenen Sprachmitteln steht für die Vorstellungsentwicklung im Vordergrund und das kann durch die Nutzung von Metaphern begünstig werden oder im Falle von nicht gut gewählten Metaphern sich negativ auf das Verstehen auswirken (Malle, 2009).

Beim Erschließen, welche Bedeutungszuschreibung eines Begriffs die richtige ist, kann der sprachliche Kontext einen Aspekt für die Textverständlichkeit darstellen (Ferstl \& d'Arcais, 1999; Underwood \& Batt, 1996). Laut Tabossi (1988) kann der sprachliche Kontext eine erleichternde Wirkung haben, wenn er die prominente Bedeutung eines Wortes stützt. Wird jedoch die ungeordnete Bedeutung durch den sprachlichen Kontext gestützt, findet eine Aktivierung beider Bedeutungen statt.

Prediger (2013b) fasst für mathematische Texte für die Wortebene Textschwierigkeiten (bzw. sprachliche Hürden) auf lexikalischer und lexikalischmorphologischer Ebene zusammen. Unter die lexikalische Ebene fallen fremde und zusammengesetzte Wörter. Die lexikalisch-morphologische Textschwierigkeit ergibt sich aus der Nutzung von Strukturwörtern, beispielsweise Präpositionen.

Auf der Ebene des Satzes ergeben sich Verknüpfungspunkte zur Wortebene durch Kohäsionsstrukturen im Text. Für die Satzebene ist die lokale Kohärenz bedeutend, die für die Mathematik insbesondere durch die Verwendung von 
relationalen Strukturwörtern hergestellt wird, u. a. die konjunktivistische und präpositionale Nutzung. Neben den direkten Beziehungen der Wort- und Satzebene durch strukturlogische Bezugssysteme ergeben sich weitere Textschwierigkeiten aufgrund von komplizierten Sätzen mit einer hohen Informationsdichte. Groeben und Christmann (1989) fassen für Textschwierigkeit auf Satzebene lange und verschachtelte Sätze, bestimmte Nebensatztypen, viele und komplexe Attribute und Funktionsverbgefüge zusammen. Jedoch können die häufig für fachliche und mathematische Fächer genannten sprachlichen Schwierigkeiten durch Passivsätze im Vergleich zu Aktiv-deklarativ-Sätzen sowie Nominalisierungen nicht per se als schwieriger betrachtet werden.

Nach Prediger (2013b) sind spezifische Sprachmittel auf Satzebene bedeutsam, die eng mit den lexikalisch-morphologischen Aspekten auf der Wortebene zusammenhängen und notwendig sind, um sinnvolle Satzstrukturen nachzuvollziehen.

Für die Textebene kann die Regelhaftigkeit von einzelnen Textsorten einen Einfluss auf die Textverständlichkeit haben. Textsorten zeichnen sich durch einen wiederkehrenden Aufbau und Strukturen aus (Artelt et al., 2007). Nach Artel et al. (2007) und Prediger (2013b) können bekannte strukturelle Spezifika von bestimmten Textsorten die Textverständlichkeit vereinfachen und durch die Verwendung von bekannten Textsorten können unklare referentielle Bezüge, die auf der Wortund Satzebene erscheinen, reduziert werden.

Des Weiteren ist auf Textebene die Bildung eines Situationsmodells eine weitere Komponente, die einen Einfluss haben kann. Wie in Abschnitt 5.2.3 erläutert, ist für Modellierungsaufgaben die Entwicklung eines Situationsmodells entscheidend, da sich durch eine geringe Textverständlichkeit Schwierigkeiten im Aufbau eines Situationsmodells und in der konzeptuellen Vorstellungen ergeben können und dies Einfluss auf folgende Teilprozesse der Modellierung haben kann (Leiss et al., 2010, 2019; Prediger, 2013b; Schukajlow \& Leiss, 2011). Die Problematik bei Modellierungsaufgaben soll nachfolgend an einem Beispiel verdeutlicht werden.

Bombenfund: Evakuierung in Brilon

Brilon. Am Mittwoch wurde in der Stadt Brilon eine Bombe aus dem Zweiten Weltkrieg gefunden. Der Blindgänger sollte, wie die Stadtverwaltung mitteilte, noch am Nachmittag entschärft werden. Alle Anwohner in einem Radius von 2.000 Metern rund um den Fundort wurden aufgefordert, das Gebiet zu verlassen. Von der Evakuierung waren auch eine Grundschule und zwei Kindergärten betroffen. In einem nahe gelegenen Schulzentrum wurde für die Anwohner ein Evakuierungszentrum eingerichtet. Turnhallen werden oftmals als Notunterkünfte vorgesehen, in denen Menschen auch 
mehrere Tage zubringen müssen. Wie viele Menschen können in der Turnhalle deiner Schule zum Übernachten als Notunterkunft untergebracht werden? Stelle deine Überlegungen übersichtlich dar (Kleine, 2012, S. 30).

Gemäß Kleine (2012) können Textschwierigkeiten, die den Verstehensprozess behindern, insbesondere aufgrund der semantischen Bedeutung von Blindgängern und Evakuierungen vorkommen. Die Schwierigkeiten können sich jedoch aufgrund von kontextspezifischen Merkmalen reduzieren. Ebenfalls wird auf die adäquate Ausbildung eines mentalen Modells (Situationsmodell) referiert: „Beispielsweise kann man eine verschlammte und verrostete Rohrbombe vor Augen haben $[\ldots]$ “ und ,[...] ein Bild vom Zustand und dem Aussehen einer solchen Turnhalle vor dem inneren Auge vorhanden“ (Kleine, 2012, S. 30).

Das Beispiel verdeutlicht, dass bei der Betrachtung von Mathematikaufgaben die Wort-, Satz- und Textebene betrachtet werden müssen, um gänzlich nachzuvollziehen, welche Textschwierigkeiten in der Formulierung einer Aufgabe vorhanden sein können.

Resümee (Abschnitt 5.2): Im Zusammenhang mit dem Begriff des Textverstehens werden vielfach weitere Begriffe verwendet, die den Begriff differenzierter für die Analyse betrachten oder Ergänzungen sind. Zur Deutung des Phänomens des Textverstehens lassen sich zwei Analyseformen unterscheiden. Das Textverstehen kann als Textrepräsentation betrachtet werden, für die die Bildung von mentalen Modellen entscheidend ist. Außerdem kann das Textverstehen unter einer Textprozessebene betrachtet werden mit Fokussierung auf die Analyse von sprachlichen Merkmalen. Erklärungsansätze verdeutlichen den Einfluss von sprachlichen Merkmalen auf die Textverständlichkeit und werden in die Betrachtung von Sprache und Text im Mathematikunterricht einbezogen.

Wenn Erkenntnisse zum Einfluss einzelner sprachlicher Merkmale existieren, stellt sich die Frage: Kann die Textschwierigkeit gemessen und vorhergesagt werden? Dieser Frage wird im nachfolgenden Kapitel nachgegangen.

\subsection{Messung und Vorhersage von Textschwierigkeit}

Im Bereich der Messung und der Vorhersage der Textschwierigkeit werden Ansätze unterschieden, die zum einen das Ziel haben, die Lesbarkeit eines Textes vorherzusagen (,prediction research ${ }^{\circ}$ ) und zum anderen, Texte zu produzieren, die lesbarer sind (,production research') (Klare, 1984, S. 703). 
Überblick (Abschnitt 5.3): Vor dem Hintergrund der Vorhersage von lesbaren Texten sind Ansätze der Lesbarkeitsforschung subsummiert, die über Kennwerte die Verständlichkeit eines Textes indizieren (Abschnitt 5.3.1). Forschungsrichtungen, die die Produktion von lesbaren Texten betrachten, sind dimensionale und interaktionale Ansätze (Abschnitt 5.3.2). Für fachliche Texte ergeben sich aufgrund von fachlichen Kriterien, die im Spannungsfeld der Erkenntnisse verständlicher Texte stehen, besondere Herausforderungen, Texte verständlich zu formulieren (Abschnitt 5.3.3). Aus den Erkenntnissen der Messung und Vorhersage der Textschwierigkeit ergeben sich grundlegende Textgestaltungs- und Optimierungsmöglichkeiten, die angesichts der Textverständlichkeit zu beachten sind (Abschnitt 5.4.3).

\subsubsection{Lesbarkeitsforschung}

Die Lesbarkeitsforschung kann als erster Ansatzpunkt zur Forschung und als empirisch-deduktiver Weg zur Bestimmung der Verständlichkeit von Texten durch Textmerkmale betrachtet werden (Groeben, 1982). Gemäß Heringer (1979) erzeugt die Lesbarkeitsforschung durch die repräsentierten Textmerkmale als Oberflächenstrukturen eine Vorhersage der Lesbarkeit eines Textes. Die Lesbarkeitsforschung vernachlässigt in allen Formen die inhaltlichen Aspekte des Textes, sei es die Art der Anschauung, die strukturelle Organisation oder weitere nichttextuelle Merkmale (Groeben, 1982). Als Textmerkmale der Lesbarkeitsforschung werden insbesondere die Wort- und Satzschwierigkeit sowie die Worthäufigkeit als Indikatoren für die Textschwierigkeit verwendet. Außerdem existiert eine Reihe weiterer Textmerkmale, die in neueren Varianten von Lesbarkeitsformeln Verwendung finden. Die Möglichkeiten des Einbezugs von Textmerkmalen umfassen die durchschnittliche Satzlänge in Wörtern, die durchschnittliche Wortlänge in Silben, die durchschnittliche Wortlänge in Buchstaben, den Prozentsatz an Präpositionen, die Anzahl der Adjektiven geteilt durch die Anzahl der Wörter, den Anteil langer Wörter oder den Anteil an Substantiven (Groeben \& Christmann, 1989; Klare, 1984; Rost, 2018).

Die Betrachtung der Annahme dieser Textmerkmale lässt sich aus den Erörterungen von Abschnitt 5.3.2 erklären. Neben den deduktiv bezogenen Textmerkmalen, die in Formeln einbezogen sind, werden Außenkriterien herangezogen, um die Formeln zu validieren. Diese Außenkriterien sind beispielsweise Expertenschätzungen, die Lesegeschwindigkeit, Behaltensleistungen, die Lesehäufigkeit und Ergänzungen im Lückentext. 
Im Verlauf des Lesbarkeitsansatzes haben sich unterschiedliche Varianten an Lesbarkeitsformeln entwickelt, beispielsweise die angepasste Flesch-Formel, der Kölner Verständlichkeitsindex für Sprache (KVIS), die erste bis dritte Wiener Sachtextformel oder der Lesbarkeitsindex (Lix) (Amstad, 1978; Bamberger \& Vanecek, 1984; Björnsson, 1968; Jussen, 1983). Die bekanntesten Lesbarkeitsformeln, der Flesch-Reading-Ease und die Adaption, nutzen zur Bestimmung der Textschwierigkeit nur die beiden Variablen durchschnittliche Satzlänge in Wörtern und durchschnittliche Wortlänge in Silben (Amstad, 1978; Flesch, 1948).

Neben diesen Lesbarkeitsformeln existieren im Bereich der Lesbarkeitsforschung weitere Ansätze zur Bestimmung der Textverständlichkeit, die nicht die Häufigkeiten von sprachlichen Merkmalen betrachten, sondern lexikalischmorphologische Varianten nutzen, um einen Indikator zu entwickeln. So können weitere Indikatoren für die Textschwierigkeit wie die lexikalische Dichte, die lexikalische Varianz, die Type-Token-Ratio oder weitere Varianten wie die Measure of Textual Lexical Diversity (MTLD) berechnet werden (Fergadiots et al., 2013; W. Johnson, 1944; Koizumi \& In'nami, 2012; Malvern \& Richards, 1997; McCarthy \& Jarvis, 2010).

Laut Groeben (1982) kann die Lesbarkeitsforschung als erschöpfend und abgeschlossen angesehen werden. Es werden zwar kaum neue Erkenntnisse aus dieser Forschungsrichtung erwartet, jedoch können die Ergebnisse als gesichert betrachtet werden, mit der Einschränkung, dass keine inhaltlichen und personenbezogene Kriterien von Texten einbezogen werden. Entsprechend werden die Textmerkmale, die die Lesbarkeitsforschung als relevante Merkmale betrachtet, in die Konstruktion von verständlichen Texten mit einbezogen. Es sollten ein geläufiger Wortschatz und eher kurze Wörter (nach Buchstaben und/oder Satzlänge) und Wörter genutzt werden, die der Schriftsprache und nicht der mündlichen Sprache (insbesondere gekennzeichnet durch Füllwörter) angehören. Vermieden werden sollten Fremdwörter. Hinsichtlich der Satzschwierigkeit sollte auf kurze und grammatikalisch einfache Sätze geachtet werden. So sind Satzschachtelungen beispielsweise bei untergeordneten Nebensätzen zu vermeiden (Groeben, 1982; Heringer, 1979).

\subsubsection{Dimensionale und prozedurale Ansätze von Verständlichkeitskonzepten}

Im Gegensatz zur Lesbarkeitsforschung, die in Abschnitt 5.3.1 dargestellt wurde, verstehen sich dimensionale Ansätze zur Ermittlung der Textverständlichkeit meist als empirisch induktiver oder theoretisch deduktiver Weg (Groeben \& 
Christmann, 1989). Es werden in der Regel drei unterschiedliche Konzeptualisierungen der dimensionalen Ansätze unterschieden: erstens der dimensionale Ansatz von Langer et al. (1974), zweitens der interaktionale Ansatz von Groeben (1982) und drittens der prozedurale Ansatz von Kintsch und Vipond (1979). Nachfolgend sollen die einzelnen Ansätze beschrieben werden.

Beim dimensionalen Ansatz von Langer et al. (1974) werden bedeutende Textmerkmale auf einer siebenstufigen Skala, die bipolar konzipiert ist, eingeschätzt. Durch faktor-analytische Verfahren wurden die Textmerkmale zu Dimensionen von Textverständlichkeit gruppiert. Die Dimensionsbeschreibung geht mit der Höhe der Ladungen, die einzelne Textmerkmale aufweisen, einher. Die Verständlichkeitsdimensionen werden dabei induktiv ermittelt. Langer et al. (1974) unterscheiden durch das Vorgehen vier Dimensionen:

1. Sprachliche Einfachheit. Mit den Merkmalen: einfache Darstellung, kurze Sätze, geläufige Wörter, Erklärung von Fachwörtern, konkret, anschaulich.

2. Gliederung und Ordnung. Mit den Merkmalen: gegliedert, folgerichtig, übersichtlich, Unterscheidung von Wesentlichem und Unwesentlichem, roter Faden erkennbar, alles kommt der Reihe nach.

3. Kürze und Prägnanz. Mit den Merkmalen: kurz, auf Wesentliche beschränkt, gedrängt, aufs Lernziel konzentriert, knapp, jedes Wort ist wichtig.

4. Zusätzliche Stimulanz. Mit den Merkmalen: anregend, interessant, abwechslungsreich, persönlich.

Die genannten Verständlichkeitsdimensionen werden über eine fünfstufige Skala durch Experten eingeschätzt, was zur Ermittlung eines quantitativen Maßstabs für die Textverständlichkeit führt. Langer et al. (1974) betrachten dabei die sprachliche Einfachheit als bedeutsamste Dimension für die Textverständlichkeit, vor der Gliederung und Ordnung. Weniger relevant für die Textverständlichkeit sind die Dimensionen Kürze und Prägnanz sowie Zusätzliche Stimulanz.

Langer et al. (1974) betrachteten für die Entwicklung der Verständlichkeitsdimensionen 66 Lehrtexte aus unterschiedlichen Bereichen. Dabei wurden auch mathematische Texte für die Untersuchung analysiert. Für die Mathematik wurden zwei Themen exemplarisch untersucht. Zum einen wurde die Einführung in die Benutzung des Rechenschiebers beim Multiplizieren am Beispiel der Aufgabe $1,5 \times 1,3$ und zum anderen eine Aufgabe zur Winkelhalbierung am Beispiel eines Winkels von $50^{\circ}$ betrachtet.

Groeben (1982) konzeptualisierte, wie im empirisch-induktiven Vorgehen des Ansatzes von Langer et al. (1974), ebenfalls Verständlichkeitsdimension. Der Unterschied des Ansatzes besteht in der Festlegung der Dimensionen auf 
theoretischer Basis, aus denen sich entsprechend Textmerkmale ableiten lassen. Aus diesem Grund wird dieser Ansatz als empirisch-deduktiv bezeichnet. Unterschieden werden bei diesem Ansatz die folgenden Dimensionen:

1. Stilistische Einfachheit. Mit den Merkmalen: kurze Satzteile, aktive Verben, aktiv-positive Formulierungen, keine Nominalisierungen, persönliche WortFormulierungen, keine Satzschachtelungen.

2. Semantische Redundanz. Mit den Merkmalen: keine wörtlichen Wiederholungen wichtiger Inhaltselemente, keine Weitschweifigkeit.

3. Kognitive Strukturierung. Mit den Merkmalen: Gebrauch von Vorstrukturierungen (advance organizer), Hervorhebung wichtiger Konzepte, sequentielles Arrangieren der Textinhalte (nach absteigendem Inklusivitätsausmaß), Zusammenfassungen, Beispielgebend, Unterschiede und Ähnlichkeiten von Konzepten verdeutlichen.

4. Konzeptueller Konflikt. Mit den Merkmalen: Neuheit und Überraschung von Konzepteigenschaften, Einfügen von inkongruenten Konzepten, alternative Problemlösungen und Fragen.

Für die Dimensionen stellt Groeben (1982) die kognitive Strukturierung als die relevanteste Dimension heraus. Weniger bedeutend ist die Stilistische Einfachheit, die zwar einen nachweisbaren, jedoch geringeren Einfluss auf die Textverständlichkeit aufweist.

Neben den zwei dimensionalen Ansätzen konzipieren Kintsch und Vipond (1979) ein Textverständlichkeitsmodell auf repräsentationaler Basis. Dabei ist der prozedurale Ansatz ebenfalls als Ergänzung zur Lesbarkeitsforschung zu betrachten und verweist explizit auf die Lesbarkeitsformeln von Flesch. Der Ansatz ist eine Erweiterung auf der Grundlage des propositionalen Ansatzes von Kintsch (1974) und dem Modell der zyklischen Textverarbeitung von Kintsch und van Dijk (1978). Der Ansatz forciert einen prozessorientierten bzw. prozeduralen Ansatz zur Erfassung der Textverständlichkeit (Grabowski, 1991). Zusammenfassend sind für diesen Ansatz drei Variablen bedeutsam: erstens die Kapazität des Kurzzeitgedächtnisses (in Propositionen), zweitens die Propositionsdichte (Wörter pro Propositionen) und drittens die Lesbarkeitswert des Textes nach der FleschFormel. Grundlegend für die Betrachtung von Propositionen sind die empirischen Ergebnisse, dass die Propositions- und Argumentationsdichte zu einer Erhöhung der Lesezeit führt und sich damit ein Einfluss auf das mentale Lexikon und das Arbeitsgedächtnis zeigt (Kintsch \& Keenan, 1973). 


\subsubsection{Textschwierigkeit von fachlichen Texten}

Texte im Fachunterricht sind geprägt durch die jeweiligen wissenschaftlichen Bezugsdisziplinen und die Verortung in der Institution Schule. Durch die funktionale Prägung von fachlichen Texten können sich Unterschiede zur Schwierigkeitsdeterminierung von fachlichen Texten ergeben.

Die von Langer et al. (1974) ermittelte Dimension Kürze und Prägnanz und die von Groeben (1982) betrachtete Dimension der semantischen Redundanz fokussieren Elemente, die sich wie in Abschnitt 4.2.5 für fachorientierte Texte als Charakteristikum ergeben. Nach Biere (2008) kann eine zusätzliche Redundanz für Texte aus dem Fachunterricht, die sich stark an der sprachlichen Gestaltung der Ursprungsdisziplin orientieren, die Textverständlichkeit erhöhen. Für die Redundanzgestaltung ist insbesondere der Satzaufbau von Bedeutung. Gemäß Groeben (1982) sollte darauf geachtet werden, dass das konzeptuell Neue der Textmitteilung am Anfang eines Satzes steht; dahinter kann Redundanz erfolgen, die Kontext öffnende Bedeutung und Vorstellungsbildung stimuliert. Für die Erstellung von Fachtexten im Unterricht und damit auch für Aufgabentexten im Mathematikunterricht ergeben sich so Implikationen, dass die durch die Sprachforschung ermittelten schwierigkeitsgenerierenden Merkmale und Dimensionen spezifisch auf den Fachunterricht bzw. auf die Mathematik bezogen werden müssen, um den Einfluss für die Texte im Fach zu klären. In Anbetracht der in Abschnitt 5.2.3 und 5.2.4 erörterten Textmerkmale, die einen negativen Einfluss auf die Textverständlichkeit haben können, sind viele Textmerkmale typisch für den fachsprachliche Gebrauch (Biere, 2008; Fraas, 2008; Oksaar, 2008; von Hahn, 2008). Die Fachsprache zeichnet sich, wie in Abschnitt 4.2.5 beschrieben, durch exklusive und zum Teil ungebräuchliche Termini, Nominalisierungen, Anonymität etc. aus. Laut Biere (2008) liegt das Prinzip der Fachsprache nicht auf der Erzeugung von Textverständlichkeit, sondern auf der Realisierung der Kommunikation innerhalb von Fachleuten über einen fachwissenschaftlichen Gegenstand unter der Prämisse, dass die Komplexität des Wirklichkeitsausschnittes bewahrt und den Ansprüchen an Fachlichkeit genügend repräsentiert wird.

Für die fachlichen Texte sind neben der Verständlichkeit des Textes auf einer kommunikativen Funktion das Verstehen der gegenstandsspezifischen Inhalte, die zu verstehen sind, auf einer kognitiven Funktion, bedeutend (vgl. Abschnitt 2.3.1). Für fachliche Texte ist die Verbindung der kommunikativen und kognitiven Funktion ein zentrales Merkmal. Biere (2008) verweist neben der sprachlichen Dekodierung des Textes auf die Kenntnis des Bezeichnungs- und Fachsystems, das durch die fachspezifischen Termini vermittelt wird. Mithin bedeutet dies, dass für die Textverständlichkeit von fachwissenschaftlich orientierten Texten die 
Bedeutung der Sache einen besonders relevanten Parameter darstellt und die Textmerkmale dahingehend nicht dieselbe Relevanz wie beispielsweise bei literarischen Texten oder Zeitungsartikeln haben. Fachtexte verständlich zu konstruieren, bedeutet entsprechend, die kognitiven und kommunikativen Voraussetzungen in individueller Weise zu betrachten. Dahingehend ergibt sich ein Spannungsfeld zwischen einerseits der fachlich präzisen sprachlichen Darstellung eines Sachverhalts und andererseits der Verständlichkeit des Textes. Die Angemessenheit der fachlichen Kommunikation ist dabei an zwei Kriterien zu bemessen, erstens der Angemessenheit der Darstellung des fachlichen Gegenstands und zweitens der Gestaltung der Textoberfläche, um diese für den Rezipienten verständlich zu halten. Dies spricht für eine spezifische adaptive Gestaltung von Texten im Mathematikunterricht. So sind Formulierungsvarianten bzw. sprachliche Variationen Möglichkeiten, die beiden Kriterien zu erfüllen.

Angesichts dessen ist für Texte im Mathematikunterricht zu klären, welchen spezifischen Einfluss sprachliche Merkmale aufweisen und in welcher Art die Merkmale verändert werden können, um Texte für Lernende anzupassen. Aus diesem Blickwinkel wird die Problematik von Abschnitt 2.4.3 von Sprache als Lernvoraussetzung und -hindernis deutlich. Textanpassung bedeutet, die Voraussetzungen für die Partizipation am Mathematikunterricht zu schaffen.

\subsubsection{Zusammenfassung abgeleiteter Textgestaltungs- und Optimierungsprinzipien}

Durch die Diskussion von Textmerkmalen zur Messung und Vorhersage der Textverständlichkeit ergeben sich grundlegende allgemeine Prinzipien der Textgestaltung und -optimierung. Die grundlegenden Aspekte sollen mit einer kurzen Zusammenfassung abgebildet werden.

Beschaffenheit des Textes: Inhaltsorganisation/-strukturierung. Nach Artelt et al. (2007) sollte für das Lesen eines Textes im Idealfall über die Sätze hinweg eine kohärente mentale Repräsentation aufgebaut und mit dem Vorwissen des Rezipienten verbunden werden. Für das Verstehen von Modellierungsaufgaben kann dies zur Erleichterung des Aufbaus eines adäquaten Situationsmodells führen (Leiss et al., 2010). Textmerkmale können diesen Prozess positiv beeinflussen. Artelt et al. (2007) gehen davon aus, dass der Aufbau von mentalen Modellen (Situationsmodellen) durch eine kohärente Organisation des Inhalts, das aufbauende und sequenzielle Arrangieren von Textinhalten und der Aktivierung des Vorwissens begünstig werden kann. In Bezug auf die adaptive Qualität der Verbesserung 
der Textverständlichkeit durch Textmerkmale müssen sich derartige Änderungen stets auf Vorwissens- und Erwartungsstrukturen des Rezipienten beziehen. Auf die Aspekte der Kohärenz und das aufbauende und sequenzielle Arrangieren soll nachfolgend näher eingegangen werden.

Kohärente Inhaltsorganisation. Damit die Rezeption eines Textes zu keinen Schwierigkeiten führt, sollten Textstrukturen genutzt werden, die deutlich machen, welche Sätze und Textteile sich aufeinander beziehen und welche davon in einen bedeutungsvollen Zusammenhang gebracht werden sollten (Artelt et al., 2007). $\mathrm{Zu}$ unterscheiden sind die lokale und globale Ebene der Kohärenz, die bereits in Abschnitt 5.3.2 erläutert wurden (Kintsch \& van Dijk, 1978). Die lokale Kohärenz bezieht sich dabei auf Satzabschnitte, die globale Kohärenz auf die Verknüpfung von generellen Textthematiken und Textteilen (Artelt et al., 2007). Laut Artelt et al. (2007) können Kohärenzlücken entstehen, die es schwierig machen, die Beziehungen in der Rezeption des Textes zu rekonstruieren, insbesondere bei ungeübten Lesenden, wenn es in einem Text nicht gelingt, kohärente Netze zwischen Sätzen und Texteilen zu bilden.

Lokale Kohärenz: Für die lokale Kohärenz sind die in Abschnitt 5.2.2 diskutierten Aspekte relevant. Nach Artelt et al. (2007) gehören die klare Verwendung von Koreferenzen beispielsweise durch Wortwiederholungen oder Verwendung von Pronomen dazu. Daneben sind weitere grammatische Strukturen wie Konjunktionen bedeutend, um Beziehungen systematisch darzustellen. Dabei zeigte sich für kausale Verknüpfungen wie weil, deshalb und daher ein positiver Effekt auf die kognitive Verarbeitungsqualität (Artelt et al., 2007). Daneben führt die Nutzung der kausalen Verknüpfungen auch zu einer Verbesserung der Inferenzbildung, also einer Ergänzung der im Text vorhandenen Informationen durch das Aktivieren der Vorwissensbasis.

Globale Kohärenz: Die globale Kohärenz wurde bereits in Abschnitt 5.2.2 in Aspekten erörtert. Dabei steht für die Entwicklung einer globalen Kohärenz der Aufbau des Textes in sinnvolle und bedeutungserleichternde Teilstrukturen im Vordergrund (Artelt et al., 2007). Erreicht werden kann dies beispielsweise durch Topic-Indikatoren, die die Abschnitte mit einführenden Sätzen, Vergleichen, Überschriften oder Beispielen miteinander in eine systematische Beziehung bringen (Schnotz, 2005). Bei inhärent genutzten globalen Kohärenzmitteln kann das In-Beziehung-Setzen der Inhalte produktiver gelingen und besonders Lesende profitieren, die eine geringere Vorwissensbasis bei der Rezeption des Textes mitbringen, sowohl auf Basis der Inferenzbildung als auch des wortwörtlichen 
Verstehens (Artelt et al., 2007; McNamara et al., 1996). Entsprechend sollten andere Strukturen vermieden werden. Dazu gehören Gedankensprünge oder fehlende Beziehungen zwischen abstrakten und konkreten Aspekten des Textes (Artelt et al., 2007).

Sequenzielles Arrangieren und Vorwissensaktivierung. Zur Verbesserung der Kohärenz eines Textes ist das sinnvolle Sequenzieren und Arrangieren notwendig, um strukturelle Bezüge von Sätzen und Textteilen zu verdeutlichen. Dies kann mit sprachlichen Mitteln realisiert werden, beispielsweise Advance Organisizers, Überschriften und Textfragen (Groeben, 1982). In Hinblick auf eine kohärente Struktur ist weiterhin das in Abschnitt 5.3.3 beschriebene Spannungsverhältnis zwischen sachlicher Korrektheit und fachlicher Stilistik und der Textverständlichkeit für den Lesenden bedeutsam. Mathematische Texte haben, wie in Abschnitt 3.2 dargestellt, entsprechend ihrer fachlichen Zugehörigkeit ein gewisses Maß an Sequenzialität und Arrangement, das sich auch aufgrund des inhaltlichen Gegenstands ergibt. Eine beliebige Veränderung eines sequenziellen, arrangierten und Vorwissen aktivierenden Textes ist nicht immer möglich und sollte beachtet werden. Gemäß Groeben (1982) sollte darauf geachtet werden, dass bei der Wahl der Veränderungsstrategie die aktuelle Fähigkeit der Lesenden in einem Ausmaß überschritten wird, das die Lesenden aufholen können. Bei moderater Optimierung eines Fachtextes, der diesen Prinzipien genügt, ist garantiert, dass die fachliche Stilistik des Fachs ebenfalls erworben werden kann und nicht nur reduktive Strategien erfolgen, die die fachsprachliche Entwicklung unter Umständen behindern.

Bilder und Diagramme: Ein bedeutsamer Aspekt für den Mathematikunterricht ist die Nutzung von Bildern und Diagrammen (nachfolgend zusammengefasst als ikonische Darstellungen). Nach Artelt et al. (2007) werden mit der Verwendung von ikonischen Darstellungen der Aufbau und die Vernetzung einer kohärenten Textrepräsentation vereinfacht. Für Bilder zeigt sich - mit Ausnahme von Bildern, die eine dekorative Funktion haben, also keine inhaltliche Bedeutung aufweisen - ein positiver Einfluss auf Behaltenseffekte, der sich jedoch nur auf die im Bild dargestellten Inhalte bezieht und nicht auf die im Text vorhandenen Informationen (Levin, 1981; Levin et al., 1987; Levin \& Lentz, 1982). Dass Darstellungen einen positiven Effekt für das Behalten aufweisen, wird meist mit der von Paivio (1986) entwickelten Kodierungstheorie gedeutet. Gemäß Peeck (1993) verläuft die Verarbeitung der durch die Darstellungen vermittelten Inhalte dabei in zwei unterschiedlichen, jedoch in Beziehung zueinander stehenden Systemen. Unterschieden wird zwischen einem propositionalen und einem imaginären System. Durch die auf beiden Seiten stattfindende Verarbeitung kommt es zu 
einer doppelten Bearbeitung der Informationen und damit zu einer erhöhten Behaltensleistung.

Besonders interessant ist der positive Effekt von Darstellungen für die kognitive Leistungsfähigkeit sowohl auf Ebene der Darstellungen als auch der im Text vorhandenen Informationen. Diese Perspektive nimmt die Analyse der Textrepräsentation, die in Abschnitt 5.2.2 diskutiert wurde, auf, um die Erleichterungen der Verarbeitung durch Darstellungen zu erklären (van Dijk \& Kintsch, 1983). Aufgrund der multimodalen Verwendung von Text und Darstellungen ergibt sich eine stützende Repräsentation, die die Entwicklung eines adäquaten mentalen Modells bzw. Situationsmodells vereinfacht (Leiss et al., 2010; van Dijk \& Kintsch, 1983). Nach Peek (1993) können Darstellungen eine Vereinfachung der Textverständlichkeit bedeuten, wenn die genutzte Text-Bild-Relation in einem der Sache dienlichen Zusammenhang gebraucht wird, also die Passung zwischen Text und Bild als produktiv betrachtet werden kann. Neben der produktiven Verwendung von Bild-Text-Relationen, die insbesondere für den Mathematikunterricht entscheidend ist, müssen die Lernenden Kompetenzen erwerben, Darstellungen interpretieren zu können. So wurde bereits in Abschnitt 5.1 auf Newman (1986) verwiesen, die die Interpretation von Darstellungen auf der zweiten Ebene des Leseverstehens betrachtet. Nach Prediger und Wessel (2012) ist es bedeutend, Voraussetzungen zu schaffen, die Bedeutung von Darstellungsformen - besonders, wenn diese nicht intuitiv zu verstehen sind - zu klären, und die Kompetenzen aufzubauen, diese Darstellungen zu interpretieren und zu vernetzen.

Lexikalische Gestaltung, syntaktische Gestaltung: Neben den genannten Aspekten zur Verbesserung der Textverständlichkeit können ebenfalls die Gestaltung der Lexik und der Syntax einbezogen werden (Groeben, 1982). Groeben (1982) subsummiert zentrale Aspekte für die Textgestaltung. Darunter fallen Motivationskraft, Textgattung, Kommunikationsform, Thema, Sprachstil, Wortschwierigkeit, Satzschwierigkeit, Kohärenz, Nutzung von Referenzen und Vorwissen.

Resümee (Abschnitt 5.3): Zur Messung der Vorhersage von Textschwierigkeiten lassen sich zwei grundlegende Forschungsperspektiven unterscheiden. Die erste Forschungsrichtung entwickelt Indikatoren, die die Textschwierigkeit durch sprachliche Merkmale vorhersagt (prediction research). Die zweite Forschungsrichtung klassifiziert sprachliche Kriterien, die zur Produktion von verständlichen Texten genutzt werden können (production research). Die Lesbarkeitsforschung, die der ersten Forschungsrichtung angehört, verwendet als Indikator zur Bestimmung der Schwierigkeit eines Textes besonders die Satz- und Wortlänge. Es gibt eine Reihe von unterschiedlichen Indikatoren, wobei neuere Lesbarkeitsformeln auch weitere Textmerkmale in die Berechnung der Formeln einbeziehen. 
Dimensionale und interaktionale Ansätze versuchen, durch die Gruppierung von Merkmalen in Dimensionen Leitkriterien zu entwickeln, die zur Herstellung von Texten dienen, die leichter verständlich sind. Für den Mathematik- und Fachunterricht sollten die Kriterien der Messung und Vorhersage der Schwierigkeit von Texten stets in Beziehung mit dem fachlichen Inhalt gedacht werden, da es möglich ist, dass allgemeine Kriterien einer positiven Textverständlichkeit nicht auf fachliche Texte bezogen werden können. Insgesamt lassen sich aus der Messung und Vorhersage der Textschwierigkeit grundlegende Aspekte ableiten, die bei der Erstellung oder Anpassung von Text berücksichtigt werden sollten.

\subsection{Sprachliche Variationen und Textschwierigkeit}

Die Kenntnis von Textmerkmalen, die die Schwierigkeit eines Textes erhöhen können, wie in Abschnitt 5.3 erörtert, führt zur Möglichkeit der Veränderungen der Textmerkmale und zur Reduktion von antizipierten sprachlichen Hürden. Die Veränderungen der Textmerkmale können als sprachliche Variationen interpretiert werden. Die sprachlichen Variationen von Textmerkmalen sind damit ein Ansatz, die Verständlichkeit von Texten zu erhöhen.

Überblick (Abschnitt 5.4): Sprachliche Variationen werden häufig mit sprachlichen Simplifizierungsstrategien in Verbindung gebracht; daraus abgeleitet werden sprachliche Variationen meist als defensive Strategie eingeordnet (Abschnitt 5.4.1). Aus speziell didaktischen Beweggründen ein sprachliches Veränderungsinstrument zu entwickeln, bildete sich für die naturwissenschaftliche und mathematische Didaktik ein sprachliches Variationsmodell heraus, das zur Veränderung von Aufgaben dient (Abschnitt 5.4.2). Sprachliche Variationen von mathematischen Testaufgaben sollen den sprachlichen Einfluss der Testleistung in Mathematiktests reduzieren; empirische Befunde weisen auf unterschiedliche Effekte der sprachlichen Variationen hin (Abschnitt 5.4.3).

\subsubsection{Variations- und Simplifizierungsstrategien für Testaufgaben}

Strategien zur Anpassung der Textverständlichkeit an die Voraussetzungen der Lesenden werden häufig als defensive Strategien bezeichnet (Leiss \& Plath, 2020; Meyer \& Tiedemann, 2017; Prediger, 2016, 2017; Rösch \& Paetsch, 2011). Unter einer defensiven Strategie ist die Reduktion von Textmerkmalen zu verstehen, die 
als ursächlich für die Textschwierigkeit gelten können. Meyer und Prediger (2012) attestieren für defensive Strategien, dass diese für Leistungssituationen teilweise und für Lernsituationen eher ungeeignet sind. Dabei handelt es sich insbesondere um die Reduktion von Textmerkmalen, die typisch für das bildungs- und fachsprachliche Register sind. Die Parallelität von Textmerkmalen des bildungsund fachsprachlichen Registers (vgl. Abschnitt 4.2.4 und Abschnitt 4.2.5) und der Textmerkmale, die aus der Perspektive der Textverständlichkeit als schwierig erachtet werden (vgl. Abschnitt 5.3), stimmen zum Teil überein.

Bei der sprachlichen Veränderung wird angenommen, dass durch die Reduktion der Textmerkmale die Textverständlichkeit zunimmt, die Textschwierigkeit abnimmt und die Reduktion von schwierigen Textmerkmalen insgesamt zu einer höheren Wahrscheinlichkeit der Lösung führt. Dabei zeigen insbesondere Lernende, die als language learner bezeichnet werden und deren Muttersprache nicht der Schulsprache entspricht, einen deutlichen Effekt bezüglich der sprachlichen Schwierigkeit von mathematischen Testaufgaben und einer geringeren Lösungsrate der Testaufgaben (Abedi et al., 2006; Haag et al., 2013; Martiniello, 2008; Shaftel \& Belton-Kocher, 2006; Wolf \& Leon, 2009). Damit ist empirisch davon auszugehen, dass eine Beziehung zwischen schwierigkeitsgenerierenden Textmerkmalen und der Lösung der Testaufgaben existiert und diese bei Lernenden mit geringeren Sprachkompetenzen einen besonderen hohen Effekt auf die Lösungshäufigkeiten hat.

\subsubsection{Mathematikdidaktisches Modell zur Variation der Textschwierigkeit von Textaufgaben}

Die Erforschung von sprachlicher Variation wird u. a. für Mathematikaufgaben und in besonderem Maß von der interdisziplinär ausgerichteten Arbeitsgruppe Fach und Sprache verfolgt (Heine et al., 2018; Leiss et al., 2017, 2019). An der Arbeitsgruppe sind Forschende aus fünf Universitäten beteiligt. Die Arbeitsgruppe entwickelt durch ein theoretisch-deduktives Verfahren ein auf drei Dimensionen beruhendes Modell zur Veränderung der sprachlichen Komplexität von Textaufgaben (Heine et al., 2018). Gemäß Heine et al. (2018) wird aus der Forschungsliteratur das Desiderat erschlossen, dass Schwierigkeiten insbesondere in den Dimensionen der strukturellen Komplexität, der Eindeutigkeit von FormBedeutung-Beziehungen und der Frequenz verortet werden können. Dabei gilt für die strukturelle Komplexität: Je höher die strukturelle Komplexität, desto sprachlich schwieriger ist ein Text. Für die beiden anderen Dimensionen der Eindeutigkeit von Form-Bedeutung-Beziehungen und der Frequenz ergeben sich 
gegenteilige Richtungseffekte. Je höher die Eindeutigkeit von Form-BedeutungBeziehungen und der Frequenz, desto geringer ist die sprachliche Schwierigkeit. Eine Operationalisierung auf sprachlicher Ebene durch konkrete Kriterien erfolgt durch die Variation von Verben, Nomen, Adjektiven, Pronomen, Syntax und der Textebene. Daraus ergibt sich ein komplexes Modell, das zur Variation von Aufgaben in enger Übereinstimmung mit der beteiligten Fachdidaktik erfolgt. Mithilfe des Modells wurden für unterschiedliche Aufgaben drei unterschiedliche Aufgabenvariationen mit einer leichten, mittleren und hohen sprachlichen Komplexität entwickelt. Das Modell kann als erstes elaboriertes System betrachtet werden, das in enger Abstimmung mit den Fachdidaktiken entwickelt wurde und sich damit von anderen Variationsansätzen, z. B. aus der Psychologie, unterscheidet. Es kann genutzt werden, um Mathematikaufgaben sprachlich zu verändern.

\subsubsection{Empirische Befunde zur Veränderung der Textschwierigkeit durch Aufgabenvariationen}

Der Einfluss der sprachlichen Komplexität hat Effekte auf das Verstehen von Mathematikaufgaben. Der Einfluss wurde im Kontext der Validität von Testsituationen bzw. Leistungsüberprüfungen im Allgemeinen (Abedi \& Lord, 2001; Wolf et al., 2008) sowie unter Berücksichtigung von potenziellen Hilfestellungen bei Lernenden mit Zweitsprache im Speziellen untersucht, um die mathematische Leistungsüberprüfung valide zu bestimmen (Abedi et al., 2004; E. Johnson \& Monroe, 2004; Sato et al., 2010).

Die Vereinfachung bzw. Simplifizierung der Sprache in einer mathematischen (Test-)Textaufgabe bzw. in einem Testitem ist, eine Möglichkeit, die Testsituationen für Lernende mit geringen sprachlichen Fähigkeiten anzupassen. Dahingehend haben sich unterschiedliche Studien damit beschäftigt, inwieweit eine Strategie der Vereinfachung von Mathematikaufgaben erfolgsversprechend ist. Einige Studien konzentrierten sich auf Analysen bezüglich der unterschiedlichen Wirkung von Items durch bestimmte Textmerkmale (Haag et al., 2013; Shaftel \& Belton-Kocher, 2006; Wolf \& Leon, 2009). Es zeigt sich für einige Textmerkmale ein Bezug zu spezifischen Registern. Shaftel et al. (2006) untersuchten schulstufenübergreifend den Einfluss von mathematischem Vokabular und konnten einen Effekt des mathematischen Vokabulars auf die Leistung für Lernende aller Schulstufen feststellen. In Kontrast dazu konnten Haag et al. (2013) keinen Effekt des mathematischen Vokabulars auf die Schwierigkeit von Testitems zeigen, für die lexikalische Textmerkmale der akademischen Sprache jedoch eine schwierigkeitsgenerierende Wirkung. Die Studien weisen daraufhin, dass 
nicht generell von einer Reduktion von theoretisch schwierig angenommenen Textmerkmalen mit einer Erhöhung der Lösungshäufigkeit von mathematischen Testitems ausgegangen werden kann. Jedoch müssen die Ergebnisse der Analyse der differenziellen Wirkung von Items durch spezifische Textmerkmale insgesamt als limitiert betrachtet werden, da durch sie keine verallgemeinernden Aussagen getroffen werden können, da die Textmerkmale in den Untersuchungen nicht systematisch variiert wurden und damit in unterschiedlicher Weise vorkommen können.

Möglichkeiten, die sprachlichen Merkmale systematisch zu variieren, sind beispielsweise das in Abschnitt 5.4.2 erwähnte Variationsmodell und andere nicht in besonderer Weise genannten Modelle zur Veränderung, die in den nachfolgenden Studien verwendet wurden.

Für die Wirksamkeit von Simplifizierungen von Textaufgaben zeigen sich unterschiedliche Effekte. So konnten Abedi et al. (2001) und Hofstetter (2003) zeigen, dass eine Vereinfachung von Textaufgaben durch Reduktion von bestimmten Textmerkmalen einen Effekt auf die Lösungshäufigkeit hat. E. Johnson und Monroe (2004) berichten hingegen, dass keine generellen positiven Effekte auf die Lösungshäufigkeit durch sprachliche Veränderung erreicht werden können. Im deutschsprachigen Raum wurde durch Haag et al. (2015) die größte angelegte Studie zur Vereinfachung von mathematischen Testaufgaben durchgeführt. In der Studie konnten keine generellen positiven Effekte bezüglich einer Vereinfachung der Testaufgaben für Lernende beobachtet werden. Es konnte jedoch gezeigt werden, dass Lernende mit Deutsch als Zweitsprache und mit einem geringen sozioökonomischen Hintergrund und geringen Leseleistungen von den Veränderungen profitieren können. Leiss et al. (2017) orientierten sich am später weiterentwickelten Modell zur sprachlichen Variation, das in Abschnitt 5.4.2 dargestellt wurde, und variierten mathematische Testaufgaben in eine sprachlich einfache, mittlere und komplexe Aufgabenvariante. In der Studie konnten keine erhöhten Lösungshäufigkeiten bei den vereinfachten Aufgaben festgestellt werden. Es zeigte sich, dass die mittelschwierigen Aufgaben die höchste Lösungshäufigkeit besitzen. Speziell für Modellierungsaufgaben konnten Plath und Leiss (2018) robuste Beziehungen zwischen sprachlichen Fähigkeiten und dem mathematischen Modellierungsprozess feststellen und außerdem konstatieren, dass bei zunehmender sprachlicher Komplexität von mathematischen Modellierungsaufgaben die Lösungshäufigkeit sinkt. 


\subsection{Desiderat}

Unter Betrachtung der Textverständlichkeit, der Merkmale für Textverständlichkeit und der Modelle und empirischen Studien zur Veränderung von mathematischen Testaufgaben ergeben sich offene Fragestellungen, die den Fokus der Arbeit zur Entwicklung eines Instruments zur sprachlichen Variation motivieren.

Offen ist, inwieweit es produktiv ist, für die theoretische Beschreibung und die empirische Untersuchung einen Zusammenhang darzustellen zwischen sprachlichen Variationen unter der in Kapitel 3 und 4 geschilderten theoretischen Fundierung und der Textoptimierung durch Messung und Vorhersage der Textverständlichkeit, die in Kapitel 5 abgebildet wurde. Diese Zusammenführung ist mit den von Groeben (1982) eingeführten differenzierten Anpassungsmöglichkeiten von Text zu assoziieren, besonders in Hinblick auf die Anpassung von Text an den Lesenden. Die Anpassung des Textes stellt eine sprachliche Variation dar, die jedoch nicht natürlich durch den Sprachgebrauch entwickelt ist, sondern durch eine technische Bearbeitung des Textes, die zu einer künstlichen sprachlichen Variation führt.

Weder bei den Analysen der differenziellen Wirkung von Items noch bei den systematischen Veränderungen von mathematischen Testaufgaben konnte eine eindeutige Wirkung von Simplifizierungsstrategien zur Vereinfachung von mathematischen Testaufgaben gezeigt werden. Hintergrund könnte sein, dass Textmerkmale eine so geringe Bedeutung für den Lösungsprozess haben, dass eine sprachliche Vereinfachung und damit die Textverständlichkeit keine Relevanz für den weiteren Verstehensprozess haben. Dagegen spricht jedoch die Studie von Plath und Leiss (2018), die darauf hinweist, dass sprachliche Variationen dann von Bedeutung sind, wenn die Sprache selbst bei Mathematikaufgaben eine hohe Relevanz besitzt, z. B. bei Modellierungsaufgaben. Darauf deuten auch die Ergebnisse von Ufer et al. (2013) hin, die die Bedeutsamkeit der Sprache je nach Aufgabenfacette betrachten und für die die Bedeutung der Sprache beispielsweise für Sachaufgaben hoch ausgeprägt sein kann. Entsprechend ist davon auszugehen, dass für eine erfolgreiche sprachliche Variation, besonders in Hinblick auf die Simplifizierung der Textmerkmale, sowohl der Aufgabentyp als auch das Instrument zur sprachlichen Variation in enger Abstimmung miteinander konzeptualisiert werden müssen. Eine solche Entwicklung eines Instruments muss die natürlichen sprachlichen Variationen, die in Textaufgaben vorkommen, als Ausgangspunkt für die Herstellung von Variationsfaktoren machen.

Das in Abschnitt 5.4.2 dargestellte sprachliche Variationsmodell von Heine et al. (2018) bietet einen Hinweis, wie die Entwicklung eines Instruments verlaufen kann. Dabei orientiert sich das Vorgehen von Heine et al. (2018) an der 
von Groeben (1982) entwickelten Verständlichkeitsdimension in Abschnitt 5.3.2. Grundlage ist bei beiden Modellen ein deduktiv-empirisches Vorgehen, das bedeutet, die Herstellung von theoretischen Dimensionen, die die Schwierigkeit abbilden, mit einer anschließenden Evaluation der hergestellten Dimensionen. Noch nicht verfolgt wurde ein an Langer et al. (1974), dargestellt in Abschnitt 5.3.2, orientiertes Vorgehen, das mit den Ansätzen von Biber (2006), erörtert in Abschnitt 4.3, und seiner theoretischen Beschreibung von Variationsdimensionen zusammenhängt. Unabhängig von der unterschiedlichen Fokussierung und Ausformulierung beider Ansätze wird ein induktiv-empirisches Vorgehen verwendet, um Faktoren zu ermitteln, bei denen Textmerkmale eine systematische Variation zeigen. Ein empirisch-induktives Vorgehen zur Konzeptualisierung des Instruments zur sprachlichen Variation könnte das Potenzial haben, die natürlichen sprachlichen Variationen in mathematischen Textaufgaben zu replizieren und als Grundlage für sprachliche Veränderungen zu machen.

Ein sprachliches Variationsinstrument, das den genannten Kriterien genügt, dient dabei nicht nur einer defensiven Strategie oder für Leistungssituationen. Durch die Betrachtung von Mathematikaufgaben, denen Lernende im Mathematikunterricht begegnen, werden sprachliche Variationsmuster transparent, die Lernende bei der Bearbeitung unterschiedlicher Mathematikaufgaben beherrschen müssen, und damit auch die sprachlichen Fähigkeiten, die für die Bearbeitung erforderlich sind. Damit ergeben sich direkte Übertragungsmöglichkeiten für die Praxis, die z. B. zur Unterstützung der Formulierung von sprachlichen Lernzielen im Zusammenhang mit fachlichen Lernzielen dienen können.

Insgesamt ergibt sich aus den offenen Fragen die Motivation, ein sprachliches Variationsinstrument $\mathrm{zu}$ entwickeln, das die sprachlichen Variationen von mathematischen Textaufgaben darstellt, die sprachliche Schwierigkeit abbildet, die fachlichen Spezifika in der Veränderung betrachtet und dazu dient, besonders Lernenden mit geringen sprachlichen Kompetenzen beim Verstehen von Textaufgaben eine Unterstützung zu bieten.

\subsection{Zusammenfassung}

Es existieren allgemeine sprachliche Anforderungen im Mathematikunterricht. Diese zeigen, dass das Textverstehen ein bedeutendes Element der Sprache im Mathematikunterricht darstellt. Das Textverstehen steht in vielfältiger Weise mit unterschiedlichen Begriffen in Verbindung, die als Differenzierungen des Begriffs Textverstehen dienen, beispielsweise die Textverständlichkeit. 
Textverstehen lässt sich auf unterschiedlichen Ebenen beschreiben. Die Ebene der Textrepräsentation forciert die Betrachtung von mentalen Modellen zur Erklärung des Textverstehens. Die Ebene des Textprozesses betrachtet hingegen die Textmerkmale, die im Text vorhanden sind und einen Einfluss auf das Textverstehen haben können. Textmerkmale haben verschiedene Einflüsse auf die Schwierigkeit eines Textes und lassen sich auch für Texte aus dem Mathematikunterricht zurückführen. In Hinblick auf das Textverstehen existieren unterschiedliche Ansätze zur Messung und Vorhersage der Schwierigkeit eines Textes. Die Lesbarkeitsforschung fokussiert Ansätze, die durch sprachliche Merkmale eine Formel erstellen, mit der ein Index für die Verständlichkeit eines Textes berechnet werden kann. Dimensionale und interaktionale Ansätze betrachten die Produktion von verständlichen Texten. Maßgebend ist die Herstellung von Verständlichkeitsdimensionen, die Textmerkmale subsummieren, die für einen verständlichen bzw. unverständlichen Text charakteristisch sind. Unter Betrachtung der unterschiedlichen Erkenntnisse der Messung und Vorhersage von Textverständlichkeit können sich unterschiedliche Effekte für fachliche bzw. mathematische Texte äußern. So kann beispielsweise die Redundanz in fachlichen Texte für eine Zunahme der Textverständlich führen.

Der Einsatz von sprachlichen Variationen zur Veränderung von mathematischen Testitems wurde zur Reduktion von sprachlichen Fehlern bei der Erhebung von mathematischen Leistungssituationen verwendet. Daneben dient die sprachliche Variation als Hilfe für Lernende mit geringen sprachlichen Fähigkeiten in mathematischen Leistungssituationen. Für die Mathematikdidaktik existiert ein sprachliches Variationsmodell, das die systematischen sprachlichen Veränderungen von Mathematikaufgaben ermöglicht. Das Modell basiert auf drei Dimensionen, in denen unterschiedliche Textmerkmale verändert werden können. Empirisch zeigt sich kein genereller Erfolg von sprachlichen Variationen, weder für eine unsystematische Betrachtung der differenziellen Wirkung von Mathematikitems noch für eine systematische Veränderung von Items durch unterschiedliche Variationsmodelle.

Aus den theoretischen und empirischen Erkenntnissen der Forschung zum Textverstehen und zur Veränderung von Mathematikaufgaben ergeben sich besonders in Hinblick auf weitere in Kapitel 3 und 4 beschriebene theoretische Begriffe offene Fragestellungen, die die Zielsetzung dieser Arbeit - die Entwicklung eines Instruments zur sprachlichen Variation von Textaufgaben - motivieren.

Ausblick: Im anschließenden empirischen Teil werden in Kapitel 6 die Zielsetzung und die Fragestellungen, die sich aus dem Erkenntnisinteresse ableiten, beschrieben. 
Open Access Dieses Kapitel wird unter der Creative Commons Namensnennung 4.0 International Lizenz (http://creativecommons.org/licenses/by/4.0/deed.de) veröffentlicht, welche die Nutzung, Vervielfältigung, Bearbeitung, Verbreitung und Wiedergabe in jeglichem Medium und Format erlaubt, sofern Sie den/die ursprünglichen Autor(en) und die Quelle ordnungsgemäß nennen, einen Link zur Creative Commons Lizenz beifügen und angeben, ob Änderungen vorgenommen wurden.

Die in diesem Kapitel enthaltenen Bilder und sonstiges Drittmaterial unterliegen ebenfalls der genannten Creative Commons Lizenz, sofern sich aus der Abbildungslegende nichts anderes ergibt. Sofern das betreffende Material nicht unter der genannten Creative Commons Lizenz steht und die betreffende Handlung nicht nach gesetzlichen Vorschriften erlaubt ist, ist für die oben aufgeführten Weiterverwendungen des Materials die Einwilligung des jeweiligen Rechteinhabers einzuholen. 